\title{
Electromagnetic Tracker Measurement Error Simulation and Tool Design
}

\author{
Gregory S. Fischer and Russell H. Taylor \\ Johns Hopkins University, CISST ERC, Baltimore, Maryland, USA \\ \{gfisch, rht\}@jhu.edu \\ http: //www.cisst.org
}

\begin{abstract}
Developing electromagnetically (EM) tracked tools can be very time consuming. Tool design traditionally takes many iterations, each of which requires construction of a physical tool and performing lengthy experiments. We propose a simulator that allows tools to be virtually designed and tested before ever being physically built. Both tool rigid body (RB) configurations and reference RB configurations are configured; the reference RB can be located anywhere in the field, and the tool is virtually moved around the reference in userspecified pattern. Sensor measurements of both RBs are artificially distorted according to a previously acquired error field mapping, and the 6-DOF frames of the Tool and Reference are refit to the distorted sensors. It is possible to predict the tool tip registration error for a particular tool and coordinate reference frame $(\mathrm{CRF})$ in a particular scenario before ever even building the tools.
\end{abstract}

\section{Introduction}

Optimal design of new electromagnetically (EM) tracked tools requires determining the quantity, relative position, and pose of sensors on tools and the corresponding coordinate reference frames (CRFs). Design is a tedious and time consuming process; to optimize a tool experimentally it takes many design iterations; for each it is necessary to build the tool, collect data, and perform error analysis. We propose a simulator that allows arbitrary tool rigid body (RB) configurations and arbitrary CRF configurations of any number of sensors to be virtually positioned in user-specified patterns and distorted according to a model of a previously acquired measurement distortion error map. This predicts the tool tip registration error for a particular tool with respect to a patient-mounted CRF in a particular scenario before ever building the physical tools.

This work spans two distinct fields related to Image Guided Surgery (IGS). First is tool design and optimization. There appears to be no analytical work directly related to EM tool design, but there has been in depth analysis of optically tracked tool design. The primary work in this field is presented in a series of papers by Fitzpatrick, West, and Maurer, the most recent of which being [1] and [2]. The second key field is EM tracker characterization and calibration, where there has been much work so this summary is far from exhaustive. Tracker characterization involves measurement of the tracking errors with respect to a ground truth reference; recent work describing this can be found in [3] and [4]. Calibration, which takes characterization information to make a model of the measurement distortion has been presented in many papers, including [5] and [6]. In this work, we adapted the formulation of [7], which specifically modeled Aurora tracker's distortion. 


\section{Measurement Error Modeling}

It is important to be able to map the measurement error in a distorted measurement field of an EM tracker; such a mapping is essential for understanding how error is affected by the environment and for modeling the distortion and further error analysis.

\subsection{Measurement Error Assessment}

Sensor measurement distortion is assessed by collecting a large quantity of measurements from the EM tracker along with corresponding reference measures from a ground truth. In our trials, the NDI Optotrak optical tracking system (OTS) is used as a reference for the NDI Aurora EM tracking system (EMTS); the Optotrak has an accuracy that is about one order of magnitude better than that of the Aurora and is effectively immune to field distortion. The EM sensors are moved throughout the working volume of the Aurora and simultaneously tracked with the EMTS and OTS. By registering the EM sensors to this OTS RB and optically tracking Aurora, it is possible to know the ground truth position and orientation (pose) of each sensor. Reference measurements are then compared to EMTS measurements for the same time step to obtain the measurement error's position and orientation components. Position error is simply the translation required to align the ideal reference position to the distorted EMTS position. Orientation error is defined as a Rodriguez vector that corresponds to the magnitude, $\theta$, and axis of rotation, $\vec{\omega}$, required to align the OTS reference to the EMTS measurement. The errors are mathematically represented as

$$
\vec{e}_{\text {pos }}=\vec{p}_{\text {measured }}-\vec{p}_{\text {ideal }} \in \mathbb{R}^{3} \quad \text { and } \quad \vec{e}_{\text {ori }}=\vec{\omega} \theta \in \mathbb{R}^{3} .
$$

\subsection{Polynomial Modeling of Measurement Error}

The detailed error mapping is used to generate a model that estimates a sensor's measurement error at a given position and orientation in the workspace of the characterized environment. Bernstein polynomials are used as the basis for these distortion models. In general, $n^{\text {th }}$ order Bernstein polynomials are defined for $0 \leq i \leq n$ by

$$
B_{i}^{n}(x)=\left(\begin{array}{c}
n \\
i
\end{array}\right) x^{i}(1-x)^{n-i} \text {, where }\left(\begin{array}{c}
n \\
i
\end{array}\right)=\frac{n !}{i !(n-i) !} .
$$

Extending Bernstein polynomial models for measurement error in 3D measurement space where the six error values from (1) are interpolated, for each value we have

$$
e(x, y, z)=\sum_{i=0}^{n} \sum_{j=0}^{n} \sum_{k=0}^{n} c_{i, j, k}^{b} B_{i}^{n}(x) B_{j}^{n}(y) B_{k}^{n}(z) \in \mathbb{R}^{6} .
$$

The model in (3) is sufficient if the measurement error of the EM system being modeled is independent of the orientation of the sensors. However, this is not a valid assumption for many tracking system; in particular, this is not valid for the Aurora 
system and the sensor orientation must be accounted for. The following algorithm accounts for both position-related and orientation-related measurement error:

- Choose a set of basis orientations vectors for which the polynomials in (3) will be generated. They should be evenly distributed as the example shown in Fig. 1.

$\begin{array}{lll}\text { Basis orientation } & \text { vectors }(14) \text { : } \\ 1 & 0 & 0 \\ 0 & 1 & 0 \\ 0 & 0 & 1 \\ 0.57735 & 0.57735 & 0.57735 \\ -0.57735 & 0.57735 & 0.57735 \\ 0.57735 & -0.57735 & 0.57735 \\ -0.57735 & -0.57735 & 0.57735 \\ -1 & 0 & 0 \\ 0 & -1 & 0 \\ 0 & 0 & -1 \\ -0.57735 & -0.57735 & -0.57735 \\ 0.57735 & -0.57735 & -0.57735 \\ -0.57735 & 0.57735 & -0.57735 \\ 0.57735 & 0.57735 & -0.57735\end{array}$

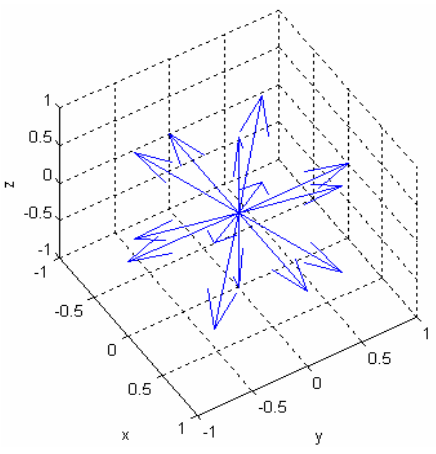

Fig. 1. Basis orientations along which distortion models are created when using 14 basis vectors. Measurements are interpolated between these to determine relative contributions of each.

- For each measurement, determine the closest three base orientation vectors that enclose the $z$-axis of the measured sensor reading, $\vec{n}_{i}$, inside of a spherical triangle defined by $\vec{b}_{1}, \vec{b}_{2}$, and $\vec{b}_{3}$ as shown in Fig. 2 . Determine the corresponding areas of each of the three spherical triangles; these that are directly proportional to the weighting of a particular base vector's contribution, $w_{i, b}$, to the error.

- Calculate the Bernstein coefficients. For each base orientation, there are six sets of coefficients in (4) to solve for: $X, Y, Z, R x, R y, R z$.

○ Normalize the measured positions to fit inside a unit cube.

- Build the six sets of equations in $A \vec{x} \approx \vec{b}$ form to solve in the least squares (LS) sense for each base vector and dimension being interpolated,

$$
\left[\begin{array}{c}
w_{1, b} e_{x}^{1} \\
\vdots \\
w_{i, b} e_{x}^{i}
\end{array}\right] \approx \beta\left[\begin{array}{c}
w_{1, b} c_{x_{0,0,0}} \\
\vdots \\
w_{i, b} c_{x_{n, n, n}}
\end{array}\right] \quad, \ldots, \quad\left[\begin{array}{c}
w_{1, b} e_{R z}^{1} \\
\vdots \\
w_{i, b} e_{R z}^{i}
\end{array}\right] \approx \beta\left[\begin{array}{c}
w_{1, b} c_{R_{0,0,0}} \\
\vdots \\
w_{i, b} c_{R_{z, n, n, n}}
\end{array}\right]
$$

Where, $w_{i, b}$ is the weight of the $\mathrm{i}^{\text {th }}$ data point for the $b^{\text {th }}$ basis vector and

$$
\beta=\left[\begin{array}{ccc}
B_{0}^{n}\left(x_{1}\right) B_{0}^{n}\left(y_{1}\right) B_{0}^{n}\left(z_{1}\right) & \cdots & B_{n}^{n}\left(x_{1}\right) B_{n}^{n}\left(y_{1}\right) B_{n}^{n}\left(z_{1}\right) \\
\vdots & & \vdots \\
B_{0}^{n}\left(x_{i}\right) B_{0}^{n}\left(y_{i}\right) B_{0}^{n}\left(z_{i}\right) & \cdots & B_{n}^{n}\left(x_{i}\right) B_{n}^{n}\left(y_{i}\right) B_{n}^{n}\left(z_{i}\right)
\end{array}\right]_{i,(n+1)^{3}} .
$$

- Solve for $6 m *(n+1)^{3}$ coefficients in the LS sense using singular value decomposition (SVD), where $m$ is the number of basis orientations. 


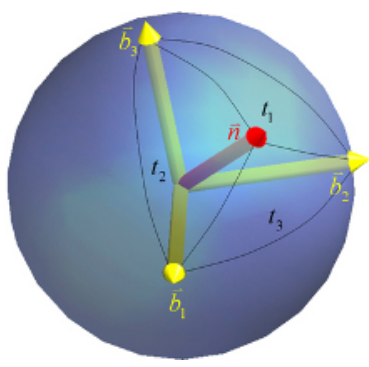

$$
\begin{array}{ll}
\vec{n} & \begin{array}{l}
\text { Measured orientation } \\
\text { (z-axis of sensor) }
\end{array} \\
\vec{b}_{1}, \vec{b}_{2}, \vec{b}_{3} \quad \begin{array}{l}
\text { Enclosing three base } \\
\text { orientation vectors }
\end{array} \\
t_{1}, t_{2}, t_{3} \quad \begin{array}{l}
\text { Areas of corresponding } \\
\text { spherical triangles } \\
\text { (normalized to sum to 1) }
\end{array}
\end{array}
$$

Fig. 2. Spherical interpolation techniques determine the relative contributions of the three closest orientations to a sensor's measurement error (and vice versa for model generation)

\section{Tool Tracking Simulation}

Contrary to the more traditional application of modeling field distortion so that measurements can be compensated in real-time (which is also a feature of our software), we are focused on simplifying tool design by removing the necessity to construct and test each physical tool in each environment of interest. The method is similar to compensation methods, but sensor measurements are artificially distorted rather than corrected.

\subsection{Calculating Measurement Distortion}

For a given position and orientation error at a given simulated sensor pose we have the distorted sensor position and orientation as

$$
\begin{gathered}
\vec{p}_{\text {distorted }}=\vec{p}_{\text {actual }}+\vec{e}_{\text {pos }} \text { and } \\
R_{\text {distorted }}=R_{\text {error }}{ }^{-1} R_{\text {actual }} \text {, where } R_{\text {error }}=e^{-\hat{\omega} \theta} \in S O(3) .
\end{gathered}
$$

Where, $\hat{\omega} \theta=\operatorname{skew}(\vec{\omega} \theta) \in \operatorname{so}(3)$ and $\vec{e}_{\text {ori }}=\bar{\omega} \theta$ is the Rodriguez vector representing the axis and angle of the estimated orientation error.

The above equations require the estimated measurement distortion for a given sensor pose, $\vec{e}_{\text {pos }}$ and $\vec{e}_{\text {ori }}$. The process for determining the error is very similar to that of generating the distortion model; the error is calculated as

$$
\vec{e}(x, y, z)=\sum_{b=1}^{3} w_{b}\left(\sum_{i=0}^{n} \sum_{j=0}^{n} \sum_{k=0}^{n} \vec{c}_{i, j, k}^{b} B_{i}^{n}(x) B_{j}^{n}(y) B_{k}^{n}(z)\right)=\left[\begin{array}{c}
\vec{e}_{\text {pos }} \\
\vec{e}_{\text {ori }}
\end{array}\right] \in \mathbb{R}^{6} .
$$

Where, the values for $b$ correspond to one of the three closest basis orientations as described earlier and $w_{b}$ corresponds to the contribution of each basis orientation. 

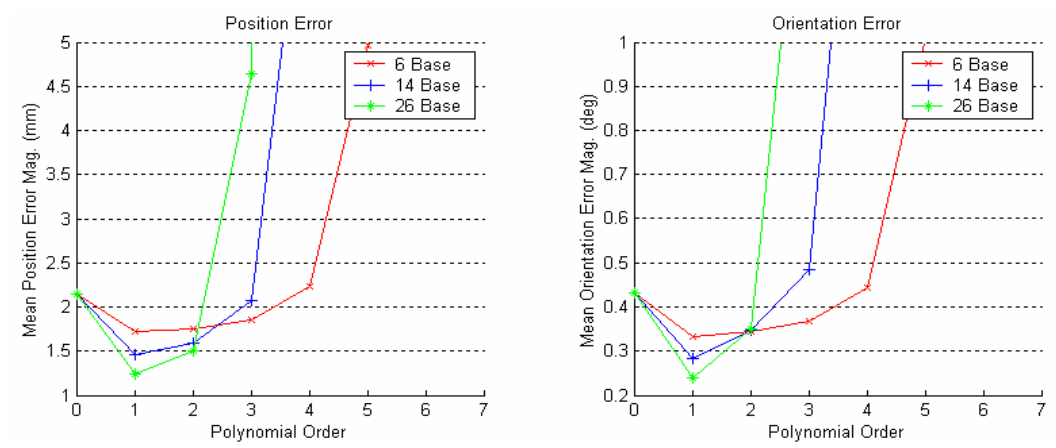

Fig. 3. Residual error for 1025 sensor measurements compensated for with a model generated an using independent data set of 1025 sensors as a function of polynomial order and angular resolution. An order of zero represents the original measurement error with no compensation.

\subsection{6-DOF Frame Fitting}

The frames $\left\{F_{1}, F_{2}, \cdots, F_{n}\right\}$ represent the known position and orientation of $n$ sensors with respect to the given $\mathrm{RB}$ frame of reference (i.e. a frame centered at a tool tip and aligned with a pointer shaft). Since sensor frames for the Aurora are only specified in $5 \mathrm{DOF}$, they can be represented as $F_{i}=\left(\vec{n}_{i}, \vec{p}_{i}\right)$.

De-meaned values of sensor positions are necessary to compute the optimal rotational alignment of an RB configuration to the corresponding measurements. These values are the measurements in the RB frame with the position of the center of gravity (CG) in the RB frame subtracted off. The best rigid point cloud to point cloud rotation that aligns the sensor RB configuration to the measurements is found in the LS sense.

Weighted orientations are treated the same way as the demeaned points; the weighting factor, $w$, keeps the position and orientation contributions balanced. The method is a modified version of that presented in [8], with the modification being the addition of orientations as just mentioned. Two variables are defined in (9); $X$ represents the configuration of the rigid body's sensors with respect to its own frame (denoted by subscript $R B$ ), and $Y$ represents the corresponding positions and orientations for the actual sensor measurements (denoted by subscript Meas).
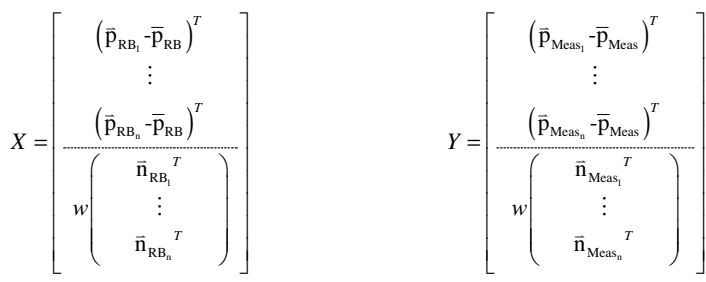

Where, $n$ represents the number of sensors, $\bar{p}$ represents the mean position of the measurement set, $\vec{n}_{i}$ represents the unit vector pointing along the $z$-axis of the given sensor, $w$ represents the weighting of the orientation measurements relative to the position measurements. This weight was analytically determined and experimentally 
confirmed to be a function of the relative position and orientation accuracy of the tracker. In the environment present in our lab, a weight of $w=100$ is satisfactory.

Using the notation in [8], two variables are defined as

$$
q_{i}=X(i, 1: 3)^{T} \quad \text { and } \quad q_{i}^{\prime}=Y(i, 1: 3)^{T} \quad \text { for } \quad i=\{1, \ldots, 2 n\} .
$$

Using the variables in (10), the matrix $H$ is calculated as

$$
H=\sum_{i=1}^{n} q_{i} q_{i}^{\prime T} .
$$

The best rigid rotation in the LS sense that aligns the tool configuration to the measurements is determined by taking the SVD of $H$ and calculating $R$ where,

$$
H=U \Lambda V^{T} \text { and } \quad R=V U^{T} .
$$

The optimal translation that aligns the RB with the measurements is then given by

$$
\vec{v}=\bar{p}_{\text {Meas }}-R * \bar{p}_{R B}
$$

\subsection{Tool Tracking}

The primary contribution of this method is that it allows for simulated tracking of a tool with respect to a reference body and determining the relative tracking error. The frame transformations of interest are detailed in Fig. 4 , the ' $\wedge$ ' and the dotted lines indicate approximate, measured transformations.

The important transformation from Fig. 4 is that of the tool with respect to the patient-fixed reference frame. It is defined as

$$
\hat{F}_{C R F}^{\text {Tool }}=\left(\hat{F}_{C R F}\right)^{-1} \hat{F}_{\text {Tool }} .
$$

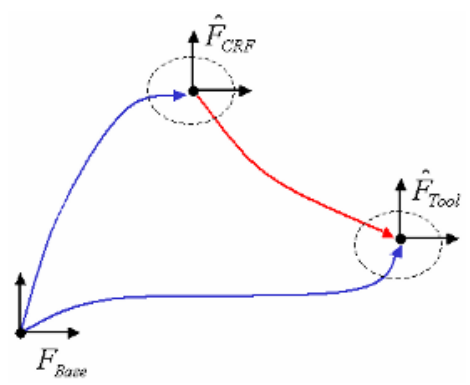

Fig. 4. Tool tracking scenario of where a tool is measured with respect to a coordinate reference frame (CRF). This is critical for IGS applications where a surgical tool is tracked with respect to a patient-fixed reference.

\section{Simulation Software}

The above algorithms have been incorporated into a single program that allows for data collection, frame fitting, real-time measurement compensation, and tool simulation; Fig. 5 displays the GUI for the simulator. The software takes a tool configuration and reference configuration from user-specified files or native NDI SROM formats. The reference is virtually placed at its commanded pose and the tool is placed with respect to the reference. Sensor locations in the base frame of the EM tracker are determined based on the RB configuration and are distorted based upon the polynomial model of the error for the chosen environment from (8). Tool RBs are refit to 
the distorted sensor readings as in (12) and (13); tracking error is the relative change in frame transformation between the reference and the tool.

The software can be run in three modes. The first mode, 'Single Point' mode, places the two RBs at the specified poses and outputs the relative tracking error. In 'Range of Motion' mode, the reference is fixed in a pose selected by the user, and the tool is moved in the specified range of positions and orientations with respect to the reference. This represents a tool being moved about a patient-fixed CRF. Finally, in 'Input Data' mode, the program accepts a text file with arbitrarily specified positions and orientations for both the tool and the reference. For all modes, summary statistics are displayed on the screen and results from each trial can be logged to file.

\subsection{Tool Design Using Simulator}

The simulator proves to be a very helpful tool for design of EM tracked instruments because a tool's performance can be gauged without ever even building it; this allows for a very large quantity of trials. In general, the tool design procedure is as follows:

1. Generate a CRF design (skip this step if one is already available)

2. Simulate the CRF design in the appropriate environment with respect to the EMTS base. Many different environments can be used for the experiments.

3. Analyze the results and decide if design satisfies requirements. If Yes, continue; if No, return to step 1.

4. Generate a Tool design and simulate with respect to the CRF.

5. Analyze the results and decide if design satisfies requirements. If Yes, continue; if No, return to step 4.

6. Build the reference and tool and compare the results.

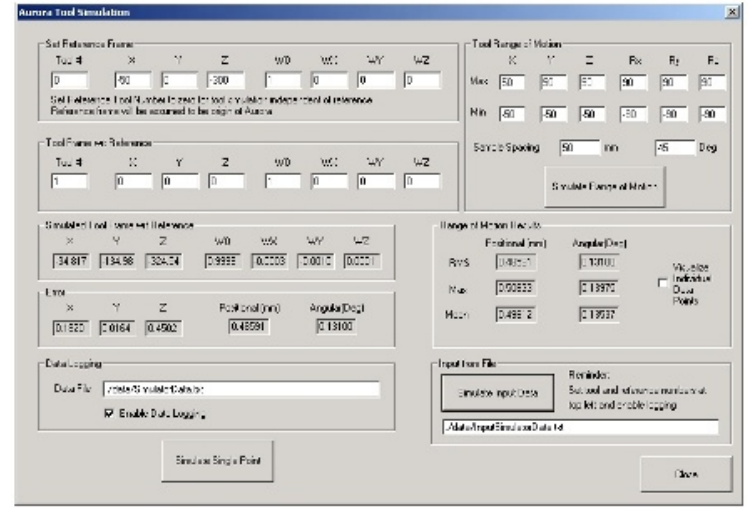

Fig. 5. GUI front end for simulation software. Allows for simulated tracking of pre-defined tools with respect to predefined references in a given characterized environment.

\section{Discussion}

A new tool for design of electromagnetically tracked instruments is presented here. It allows for rapid prototyping and design of EM tracked tools without the necessity to physically build and experiment with many different designs. This allows for the prospect of faster design of higher quality tracked instruments. Initial experiments show that the polynomial model, when used for measurement compensation, produces a very accurate representation of the data. The RMS residual error for a mildly disturbed data set that began with RMS errors of $2.30 \mathrm{~mm}$ and $0.45^{\circ}$ was below $0.20 \mathrm{~mm}$ and $0.05^{\circ}$ for a $6^{\text {th }}$ order model with 14 basis orientations. 
To get a better idea of how representative the measurement distortion model really is, it was applied to an independent set of sensor measurements with know reference measurements; the results are shown in Fig. 3. Realistically, it appears that we can expect the model to decrease the residual error by a factor of about two. Fortunately, the minimum residual error occurs at a lower order model $\left(1^{\text {st }}\right.$ or $2^{\text {nd }}$ order $)$, so the quantity of data required for generation of the model can be reduced to make collection reasonable for a given practical environment such as an operating room. The compensation results are quite good, and therefore, we can expect the simulator to produce realistic distortions since it based off of the same model. Thus far, the procedure has been used successfully to help design instruments and references for ENT surgery including a head-mounted CRF, endoscope, pointer, and tissue shaver. Further results and more detail of these methods are available in [9].

\section{Acknowledgements}

Funding for this work was provided by Northern Digital Inc., Waterloo, Canada. General infrastructure support was provided by the CISST ERC under agreement EEC-9731478. We would also like to thank Paul MacDonald, Jeff Stanly, Saibal Chakraburtty, John Niemenan, Stefan Kirsch, Gouthami Chintalapani, Xiaofeng Liu, and Katherin Peperzak for their assistance in various aspects of this work.

\section{References}

1. J. Michael Fitzpatrick and Jay B. West. The Distribution of Target Registration Error in Rigid-Body Point-Based Registration. IEEE Trans. on Med. Imaging. Vol. 20. No. 9. Sept. 2001: 917-927.

2. Jay B. West and Calvin R. Maurer, Jr. Designing Optically Tracked Instruments for ImageGuided Surgery. IEEE Trans. on Med. Imaging, Vol. 23. No. 5. May 2004: 533-545.

3. J. Hummel, M. Figl, C. Kollmann, H. Bergmann, W. Birkfellner. Evaluation of a Miniature Electromagnetic Position Tracker. Med. Physics. Oct. 2002. Vol. 29, No. 10: 2205-2212.

4. A. Wagner, K. Schicho, W. Birkfellner et. al. Quantitative Analysis of Factors Affecting Intraoperative Precision and Stability of Optoelectronic and Electromagnetic Tracking Systems. Medical Physics. May 2002. Vol. 29, No. 5: 905-912.

5. A.J. Chung, P.J. Edwards, F. Deligianni, and G. Yang. Freehand Cocalibration of Optical and Electromagnetic Trackers for Navigated Bronchoscopy. MIAR 2004. Aug. 2004, Springer LNCS 3150: 320-328.

6. K. Kanada, et. al. A Rapid Method for Magnetic Tracker Calibration Using a MagnetoOptical Hybrid Tracker. MICCAI 2003. Nov. 2003. Springer LNCS 2879: 285-293.

7. Xiaohui $\mathrm{Wu}$ and Russell Taylor. A Direction Space Interpolation Technique for Calibration of Electromagnetic Surgical Navigation Systems. MICCAI 2003. Nov. 2003. Springer LNCS 2879: 215-222.

8. K.S. Arun, T.S. Huang, and S.D. Blostein. Least-Squares Fitting of Two 3-D Point Sets. IEEE PAMI. Vol. 9. No. 5. Sept. 1987: 698-700.

9. Gregory S. Fischer. Electromagnetic Tracker Characterization and Optimal Tool Design With Applications to ENT Surgery. Masters thesis, Johns Hopkins University, Baltimore, MD, USA. May 2005. (http://www.cisst.org/ gfischer) 